\title{
Stochastic Differential Equations with Multi-Markovian Switching
}

\author{
Meng Liu ${ }^{1}$ and Ke Wang ${ }^{2}$ \\ ${ }^{1}$ School of Mathematical Science, Huaiyin Normal University, Huaian 223300, China \\ ${ }^{2}$ Department of Mathematics, Harbin Institute of Technology, Weihai 264209, China
}

Correspondence should be addressed to Meng Liu; liumeng0557@sina.com

Received 31 August 2012; Revised 2 March 2013; Accepted 5 March 2013

Academic Editor: Jose L. Gracia

Copyright (c) 2013 M. Liu and K. Wang. This is an open access article distributed under the Creative Commons Attribution License, which permits unrestricted use, distribution, and reproduction in any medium, provided the original work is properly cited.

This paper is concerned with stochastic differential equations (SDEs) with multi-Markovian switching. The existence and uniqueness of solution are investigated, and the $p$ th moment of the solution is estimated. The classical theory of SDEs with single Markovian switching is extended.

\section{Introduction}

Stochastic modeling has played an important role in many branches of industry and science. SDEs with single continuous-time Markovian chain have been used to model many practical systems where they may experience abrupt changes in their parameters and structure caused by phenomena such as abrupt environment disturbances. SDEs with single Markovian switching can be denoted by

$$
\begin{aligned}
d x(t)= & f(x(t), t, \gamma(t)) d t \\
& +g(x(t), t, \gamma(t)) d B(t), \quad t_{0} \leq t \leq T
\end{aligned}
$$

with initial conditions $x\left(t_{0}\right)=x_{0} \in L_{\mathscr{F}_{t_{0}}}^{2}$ and $\gamma\left(t_{0}\right)=\gamma_{0}$, where $\gamma(t)$ is a right-continuous homogenous Markovian chain on the probability space taking values in a finite state space $\mathbb{S}=\{1,2, \ldots, N\}$ and is $\mathscr{F}_{t}$-adapted but independent of the Brownian motion $B(t)$, and

$$
\begin{gathered}
f: \mathbb{R}^{n} \times \mathbb{R}_{+} \times \mathbb{S} \longrightarrow \mathbb{R}^{n}, \\
g: \mathbb{R}^{n} \times \mathbb{R}_{+} \times \mathbb{S} \longrightarrow \mathbb{R}^{n+m} .
\end{gathered}
$$

Owing to their theoretical and practical significance, (1) has received great attention and has been recently studied extensively, and we here mention Skorokhod [1] and Mao and Yuan [2] among many others.
However, in the real world, the condition that coefficients $f$ and $g$ in (1) are perturbed by the same Markovian chain is too restrictive. For example, in the classical Black-Scholes model, the asset price is given by a geometric Brownian motion

$$
d X(t)=\mu X(t) d t+v X(t) d B(t)
$$

where $\mu$ is the rate of the return of the underlying assert, $v$ is the volatility, and $B(t)$ is a scalar Brownian motion. Since there is strong evidence to indicate that $\mu$ is not a constant but is a Markovian jump process (see, e.g., $[3,4]$ ), many authors proposed the following model:

$$
d X(t)=\mu(\gamma(t)) X(t) d t+\nu(\gamma(t)) X(t) d B(t) .
$$

However, many stochastic factors that affect $\mu$ are different from those that affect $\nu$. Then the following model is more appropriate than model (105) to describe this problem:

$$
d X(t)=\mu\left(\gamma_{1}(t)\right) X(t) d t+\nu\left(\gamma_{2}(t)\right) X(t) d B(t),
$$

where $\gamma_{i}(t)$ is a right-continuous homogenous Markovian chain taking values in a finite state space, $i=1,2$. Another example is the stochastic Lotka-Volterra model with single Markovian switching which has received great attention and has been studied extensively recently (see, e.g., [5-12]). 
For the sake of convenience, we take the following twodimensional competitive model as an example:

$$
\begin{aligned}
d x_{1}= & x_{1}\left[r_{10}(\gamma(t))-a_{11}(\gamma(t)) x_{1}-a_{12}(\gamma(t)) x_{2}\right] d t \\
& +\alpha_{1}(\gamma(t)) x_{1} d B_{1}(t), \\
d x_{2}= & x_{2}\left[r_{20}(\gamma(t))-a_{21}(\gamma(t)) x_{1}-a_{22}(\gamma(t)) x_{2}\right] d t \\
& +\alpha_{2}(\gamma(t)) x_{2} d B_{2}(t),
\end{aligned}
$$

where $x_{i}$ is the size of $i$ th species at time $t, r_{i 0}(j)$ represents the growth rate of $i$ th species in regime $j$ for $i=1,2$, $j \in \mathbb{S}$, and $B_{1}$ and $B_{2}$ are independent standard Brownian motions. However, there are many stochastic factors that affect some coefficients intensely but have little impact on other coefficients in (6). For example, suppose that the stochastic factor is rain falls and $x_{1}$ is able to endure a damp weather while $x_{2}$ is fond of a dry environment, then the rain falls will affect $x_{2}$ intensely but have little impact on $x_{1}$. Thus, a more appropriate model is governed by

$$
\begin{aligned}
d x_{1}= & x_{1}\left[r_{10}\left(\gamma_{10}(t)\right)-a_{11}\left(\gamma_{11}(t)\right) x_{1}-a_{12}\left(\gamma_{12}(t)\right) x_{2}\right] d t \\
& +\alpha_{1}\left(\gamma_{1}(t)\right) x_{1} d B_{1}(t), \\
d x_{2}= & x_{2}\left[r_{20}\left(\gamma_{20}(t)\right)-a_{21}\left(\gamma_{21}(t)\right) x_{1}-a_{22}\left(\gamma_{22}(t)\right) x_{2}\right] d t \\
& +\alpha_{2}\left(\gamma_{2}(t)\right) x_{2} d B_{2}(t),
\end{aligned}
$$

where $\gamma_{i j}(t)$ and $\gamma_{k}(t)$ are right-continuous homogenous Markovian chains taking values in finite state spaces $\mathbb{S}_{i j}$ for $i=1,2, j=0,1,2$, and $\mathbb{S}_{k}$ for $k=1,2$, respectively.

Thus the above examples show that the study of the following SDEs with multi-Markovian switchings is essential and is of great importance from both theoretical and practical points:

$$
\begin{aligned}
d x(t)= & f\left(x(t), t, \gamma_{1}(t)\right) d t \\
& +g\left(x(t), t, \gamma_{2}(t)\right) d B(t), \quad t_{0} \leq t \leq T
\end{aligned}
$$

with initial conditions $x\left(t_{0}\right)=x_{0} \in L_{\mathscr{F}_{0}}^{2}$ and $\gamma_{i}\left(t_{0}\right)$, where $\gamma_{i}(t)$ is a right-continuous homogenous Markovian chain on the probality space taking values in a finite state space $\mathbb{S}_{i}=\left\{1,2, \ldots, N_{i}\right\}$ and is $\mathscr{F}_{t}$-adapted but independent of the Brownian motion $B_{i}(t), i=1,2$, and

$$
\begin{gathered}
f: \mathbb{R}^{n} \times \mathbb{R}_{+} \times \mathbb{S}_{1} \longrightarrow \mathbb{R}^{n}, \\
g: \mathbb{R}^{n} \times \mathbb{R}_{+} \times \mathbb{S}_{2} \longrightarrow \mathbb{R}^{n+m} .
\end{gathered}
$$

Equation (8) can be regarded as the result of the $N_{1} \times N_{2}$ equations

$$
\begin{aligned}
d x(t)= & f(x(t), t, i) d t \\
& +g(x(t), t, j) d B(t), \quad i \in \mathbb{S}_{1}, j \in \mathbb{S}_{2}
\end{aligned}
$$

switching among each other according to the movement of the Markovian chains. It is important for us to discover the properties of the system (8) and to find out whether the presence of two Markovian switchings affects some known results. The first step and the foundation of those studies are to establish the theorems for the existence and uniqueness of the solution to system (8). So in this paper, we will give some theorems for the existence and uniqueness of the solution to system (8) and study some properties of this solution. The theory developed in this paper is the foundation for further study and can be applied in many different and complicated situations, and hence the importance of the results in this paper is clear.

It should be pointed out that the theory developed in this paper can be generalized to cope with the more general SDEs with more Markovian chains

$$
\begin{aligned}
d x(t)= & f\left(x(t), t, \gamma_{1}(t), \ldots, \gamma_{n}(t)\right) d t \\
& +g\left(x(t), t, \gamma_{n+1}(t), \ldots, \gamma_{n+m}(t)\right) d B(t) .
\end{aligned}
$$

The reason we concentrate on (8) rather than (11) is to avoid the notations becoming too complicated. Once the theory developed in this paper is established, the reader should be able to cope with the more general (11) without any difficulty.

The remaining part of this paper is as follows. In Section 2, the sufficient criteria for existence and uniqueness of solution, local solution, and maximal local solution will be established, respectively. In Section 3, the $L^{P}$-estimates of the solution will be given. In Section 4, we will introduce an example to illustrate our main result. Finally, we will close the paper with conclusions in Section 5.

\section{SDEs with Markovian Chains}

Throughout this paper, let $\left(\Omega, \mathscr{F},\left\{\mathscr{F}_{t}\right\}_{t \in R_{+}}, \mathscr{P}\right)$ be a complete probability space. Let $B(t)=\left(B_{1}(t), \ldots, B_{m}(t)\right)^{T}$ be an $m$ dimensional Brownian motion defined on the probability space.

In this section, we will consider (8). Let $\gamma(t)=\left(\gamma_{1}(t)\right.$, $\left.\gamma_{2}(t)\right)$. We impose a hypothesis.

(H1): $\gamma_{1}(t)$ is independent of $\gamma_{2}(t)$.

Then $\gamma(t)$ is a homogenous vector Markovian chain with transition probabilities

$$
\begin{aligned}
& P\left\{\gamma(s+t)=\left(j_{1}, j_{2}\right) \mid \gamma(s)=\left(i_{1}, i_{2}\right)\right\} \\
& \quad=P\left\{\gamma(t)=\left(j_{1}, j_{2}\right) \mid \gamma(0)=\left(i_{1}, i_{2}\right)\right\} \\
& =: P_{i_{1} i_{2} ; j_{1} j_{2}}(t),
\end{aligned}
$$

where $\left(i_{1}, i_{2}\right),\left(j_{1}, j_{2}\right) \in \mathbb{S}=\left\{(1,1),(1,2), \ldots,\left(1, N_{2}\right),(2,1)\right.$, $\left.\ldots,\left(N_{1}, N_{2}\right)\right\}$.

Now, we will prepare some lemmas which are important for further study.

Lemma 1. $P_{i_{1} i_{2} ; j_{1} j_{2}}(t)$ has the following properties:

(i) $P_{i_{1} i_{2} ; j_{1} j_{2}}(t) \geq 0$ for $\left(i_{1}, i_{2}\right),\left(j_{1}, j_{2}\right) \in \mathbb{S}$;

(ii) $\sum_{\left(j_{1}, j_{2}\right) \in \mathbb{S}} P_{i_{1} i_{2} ; j_{1} j_{2}}(t)=1$ for $\left(i_{1}, i_{2}\right) \in \mathbb{S}$;

(iii) $P_{i_{1} i_{2} ; j_{1} j_{2}}(0)=\delta_{i_{1} j_{1}} \cdot \delta_{i_{2} j_{2}}$, where $\delta_{i_{k} j_{k}}=1$ if $i_{k}=j_{k}$, otherwise $\delta_{i_{k} j_{k}}=0, k=1,2$; 
(iv) (Chapman-Kolmogorov equation) For $s, t \geq 0$ and $\left(i_{1}, i_{2}\right),\left(j_{1}, j_{2}\right) \in \mathbb{S}$,

$$
\begin{aligned}
& P_{i_{1} i_{2} ; j_{1} j_{2}}(s+t) \\
& \quad=\sum_{\left(k_{1}, k_{2}\right) \in \mathbb{S}} P_{i_{1} i_{2} ; k_{1} k_{2}}(t) P_{k_{1} k_{2} ; j_{1} j_{2}}(s) .
\end{aligned}
$$

Proof. The proofs of (i), (ii), and (iii) are obvious. Now, let us prove (iv):

$$
\begin{aligned}
& P_{i_{1} i_{2} ; j_{1} j_{2}}(s+t) \\
& =P\left\{\gamma(s+t)=\left(j_{1}, j_{2}\right) \mid \gamma(0)=\left(i_{1}, i_{2}\right)\right\} \\
& =\sum_{\left(k_{1}, k_{2}\right) \in \mathbb{S}} P\left\{\gamma(s+t)=\left(j_{1}, j_{2}\right),\right. \\
& \left.\gamma(t)=\left(k_{1}, k_{2}\right) \mid \gamma(0)=\left(i_{1}, i_{2}\right)\right\} \\
& =\sum_{\left(k_{1}, k_{2}\right) \in \mathbb{S}}\left(P \left\{\gamma(s+t)=\left(j_{1}, j_{2}\right),\right.\right. \\
& \left.\gamma(t)=\left(k_{1}, k_{2}\right), \gamma(0)=\left(i_{1}, i_{2}\right)\right\} \\
& \left.\times\left(P\left\{\gamma(t)=\left(k_{1}, k_{2}\right), \gamma(0)=\left(i_{1}, i_{2}\right)\right\}\right)^{-1}\right) \\
& \times \frac{P\left\{\gamma(t)=\left(k_{1}, k_{2}\right), \gamma(0)=\left(i_{1}, i_{2}\right)\right\}}{P\left\{\gamma(0)=\left(i_{1}, i_{2}\right)\right\}} \\
& =\sum_{\left(k_{1}, k_{2}\right) \in \mathbb{S}} P\left\{\gamma(s+t)=\left(j_{1}, j_{2}\right) \mid \gamma(t)=\left(k_{1}, k_{2}\right)\right. \text {, } \\
& \left.\gamma(0)=\left(i_{1}, i_{2}\right)\right\} \\
& \times P\left\{\gamma(t)=\left(k_{1}, k_{2}\right) \mid \gamma(0)=\left(i_{1}, i_{2}\right)\right\} \\
& =\sum_{\left(k_{1}, k_{2}\right) \in \mathbb{S}} P\left\{\gamma(s+t)=\left(j_{1}, j_{2}\right) \mid \gamma(t)=\left(k_{1}, k_{2}\right)\right\} \\
& \times P\left\{\gamma(t)=\left(k_{1}, k_{2}\right) \mid \gamma(0)=\left(i_{1}, i_{2}\right)\right\} \\
& =\sum_{\left(k_{1}, k_{2}\right) \in \mathbb{S}} P_{k_{1} k_{2} ; j_{1} j_{2}}(s) P_{i_{1} i_{2} ; k_{1} k_{2}}(t) \text {. }
\end{aligned}
$$

This completes the proof.

Now, we impose another hypothesis, which is called standard condition.

$$
\text { (H2): } \lim _{t \rightarrow 0} P_{i_{1} i_{2} ; j_{1} j_{2}}(t)=\delta_{i_{1} i_{2} ; j_{1} j_{2}} .
$$

Lemma 2. Under Assumption (H2), for all $t \geq 0,\left(i_{1}, i_{2}\right) \in \mathbb{S}$, one has $P_{i_{1} i_{2} ; i_{1} i_{2}}(t)>0$.

Proof. From $P_{i_{1} i_{2} ; i_{1} i_{2}}(0)>0$ and (H2) we know that, for arbitrary fixed $t>0$, we have

$$
P_{i_{1} i_{2} ; i_{1} i_{2}}\left(\frac{t}{n}\right)>0
$$

for sufficient large $n$. Then making use of Chapman-Kolmogorov equation

$$
\begin{aligned}
P_{i_{1} i_{2} ; i_{1} i_{2}}(s+t) & =\sum_{\left(k_{1}, k_{2}\right) \in \mathbb{S}} P_{i_{1} i_{2} ; k_{1} 1 k_{2}}(t) P_{k_{1} k_{2} ; i_{1} i_{2}}(s) \\
& \geq P_{i_{1} i_{2} ; i_{1} i_{2}}(t) P_{i_{1} i_{2} ; i_{1} i_{2}}(s)
\end{aligned}
$$

gives

$$
P_{i_{1} i_{2} ; i_{1} i_{2}}(t) \geq\left(P_{i_{1} i_{2} ; i_{1} i_{2}}\left(\frac{t}{n}\right)\right)^{n}>0,
$$

which is the desired assertion.

Lemma 3. Under Assumption (H2), for all $\left(i_{1}, i_{2}\right) \in \mathbb{S}$,

$$
-q_{i_{1} i_{2} ; i_{1} i_{2}}:=\lim _{t \rightarrow 0} \frac{1-P_{i_{1} i_{2} ; i_{1} i_{2}}(t)}{t}
$$

exists (but may be $\infty$ ).

Proof. Define $\phi(t)=-\ln P_{i_{1} i_{2} ; i_{1} i_{2}}(t) \geq 0$. Then making use of (16) gives

$$
\phi(s+t) \leq \phi(s)+\phi(t) .
$$

Set $-q_{i_{1} i_{2} ; i_{1} i_{2}}=\sup _{t>0}(\phi(t) / t)$. It is easy to see that

$$
0 \leq-q_{i_{1} i_{2} ; i_{1} i_{2}} \leq \infty, \quad \limsup _{t \rightarrow 0} \frac{\phi(t)}{t} \leq-q_{i_{1} i_{2} ; i_{1} i_{2}} .
$$

Now we will assert

$$
\liminf _{t \rightarrow 0} \frac{\phi(t)}{t} \geq-q_{i_{1} i_{2} ; i_{1} i_{2}} .
$$

In fact, for $0<h<t, \exists n, 0 \leq \varepsilon \leq h$ such that $t=n h+\varepsilon$. Applying (19) yields

$$
\frac{\phi(t)}{t} \geq \frac{n h}{\varepsilon} \frac{\phi(h)}{h}+\frac{\phi(\varepsilon)}{t} .
$$

Note that $\varepsilon \rightarrow 0, n h / \varepsilon \rightarrow 1, \phi(\varepsilon) \rightarrow 0$ whenever $h \rightarrow 0^{+}$, then for all $t>0$ we have

$$
\frac{\phi(t)}{t} \leq \liminf _{h \rightarrow 0} \frac{\phi(h)}{h} .
$$

This implies $-q_{i_{1} i_{2} ; i_{1} i_{2}} \leq \liminf _{t \rightarrow 0}(\phi(t) / t)$. Thus

$$
-q_{i_{1} i_{2} ; i_{1} i_{2}}=\lim _{t \rightarrow 0} \frac{\phi(t)}{t} .
$$

Using the definition of $\phi(t)$ gives

$$
\lim _{t \rightarrow 0} \frac{1-P_{i_{1} i_{2} ; i_{1} i_{2}}(t)}{t}=\lim _{t \rightarrow 0} \frac{1-\exp \{\phi(t)\}}{\phi(t)} \frac{\phi(t)}{t}=-q_{i_{1} i_{2} ; i_{1} i_{2}},
$$

which is the required assertion. 
Lemma 4. Under Assumption (H2), for $\left(i_{1}, i_{2}\right),\left(j_{1}, j_{2}\right) \in$ $\mathbb{S},\left(i_{1}, i_{2}\right) \neq\left(j_{1}, j_{2}\right)$,

$$
q_{i_{1} i_{2} ; j_{1} j_{2}}:=P_{i_{1} i_{2} ; j_{1} j_{2}}^{\prime}(0)=\lim _{t \rightarrow 0} \frac{P_{i_{1} i_{2} ; j_{1} j_{2}}(t)}{t}
$$

exists and is finite.

Proof. By (H2), we note that for all $0<\varepsilon<1 / 3, \exists 0<\delta<1$, such that

$$
\begin{gathered}
P_{i_{1} i_{2} ; i_{1} i_{2}}(t)>1-\varepsilon, \quad P_{j_{1} j_{2} ; j_{1} j_{2}}(t)>1-\varepsilon, \\
P_{j_{1} j_{2} ; i_{1} i_{2}}(t)<\varepsilon
\end{gathered}
$$

provided $0<t \leq \delta$.

For $\forall 0 \leq h<t$, set $n=\langle t / h\rangle$, where $\langle a\rangle=\max _{n \leq a}\{n \in$ $\mathbb{Z}$ \}. Let

$$
\begin{gathered}
P_{i_{1} i_{2} ; k_{1} k_{2}}^{\left(j_{1} j_{2}\right)}(h)=P_{i_{1} i_{2} ; k_{1} k_{2}}(h), \\
P_{i_{1} i_{2} ; k_{1} k_{2}}^{\left(j_{1} j_{2}\right)}(m h) \\
=\sum_{\left(r_{1}, r_{2}\right) \neq\left(j_{1}, j_{2}\right)} P_{i_{1} i_{2} ; r_{1} r_{2}}^{\left(j_{1} j_{2}\right)}((m-1) h) P_{r_{1} r_{2} ; k_{1} k_{2}}(h),
\end{gathered}
$$

where $P_{i_{1} i_{2} ; k_{1} k_{2}}^{\left(j_{1} j_{2}\right)}(m h)$ means that the probability of the $\gamma(t)$ will not reach to $\left(j_{1}, j_{2}\right)$ at times $h, 2 h, \ldots,(m-1) h$ but will reach to $\left(k_{1}, k_{2}\right)$ at time $m h$. Note that if $h \leq t \leq \delta$, then

$$
\begin{aligned}
\varepsilon & >1-P_{i_{1} i_{2} ; i_{1} i_{2}}(t) \\
& =\sum_{\left(k_{1}, k_{2}\right) \neq\left(i_{1}, i_{2}\right)} P_{i_{1} i_{2} ; k_{1} k_{2}}(t) \geq P_{i_{1} i_{2} ; j_{1} j_{2}}(t) \\
& \geq \sum_{m=1}^{n} P_{i_{1} i_{2} ; j_{1} j_{2}}^{\left(j_{1} j_{2}\right)}(m h) P_{j_{1} j_{2} ; j_{1} j_{2}}(t-m h) \\
& \geq(1-\varepsilon) \sum_{m=1}^{n} P_{i_{1} i_{2} ; j_{1} j_{2}}^{\left(j_{1} j_{2}\right)}(m h),
\end{aligned}
$$

which indicates

$$
\sum_{m=1}^{n} P_{i_{1} i_{2} ; j_{1} j_{2}}^{\left(j_{1} j_{2}\right)}(m h) \leq \frac{\varepsilon}{1-\varepsilon} .
$$

Then making use of

$$
\begin{aligned}
P_{i_{1} i_{2} ; i_{1} i_{2}}(m h)= & P_{i_{1} i_{2} ; i_{1} i_{2}}^{\left(j_{1} j_{2}\right)}(m h) \\
& +\sum_{l=1}^{m-1} P_{i_{1} i_{2} j_{1} j_{2}}^{\left(j_{1} j_{2}\right)}(l h) P_{j_{1} j_{2} ; i_{1} i_{2}}((m-l) h),
\end{aligned}
$$

we obtain

$$
\begin{aligned}
P_{i_{1} i_{2} ; i_{1} i_{2}}^{\left(j_{1} j_{2}\right)}(m h) & \geq P_{i_{1} i_{2} ; i_{1} i_{2}}(m h)-\sum_{l=1}^{m-1} P_{i_{1} i_{2} ; j_{1} j_{2}}^{\left(j_{1} j_{2}\right)}(l h) \\
& \geq 1-\varepsilon-\frac{\varepsilon}{1-\varepsilon} .
\end{aligned}
$$

Consequently,

$$
\begin{aligned}
P_{i_{1} i_{2} ; j_{1} j_{2}} & (t) \\
& >\sum_{m=1}^{n} P_{i_{1} i_{2} ; i_{1} i_{2}}^{\left(j_{1} j_{2}\right)}((m-1) h) P_{i_{1} i_{2} ; j_{1} j_{2}}(h) P_{j_{1} j_{2} ; j_{1} j_{2}}(t-m h) \\
& \geq n\left(1-\varepsilon-\frac{\varepsilon}{1-\varepsilon}\right) P_{i_{1} i_{2} ; j_{1} j_{2}}(h)(1-\varepsilon) \\
& \geq n(1-3 \varepsilon) P_{i_{1} i_{2} ; j_{1} j_{2}}(h) .
\end{aligned}
$$

Dividing both sides of the above inequality by $h$ and noting $n h \rightarrow t$ whenever $h \rightarrow 0$ yield

$$
\limsup _{h \rightarrow 0} \frac{P_{i_{1} i_{2} ; j_{1} j_{2}}(h)}{h} \leq \frac{1}{1-3 \varepsilon} \frac{P_{i_{1} i_{2} ; j_{1} j_{2}}(t)}{t}<\infty .
$$

Then letting $t \rightarrow 0$ gives

$$
\limsup _{h \rightarrow 0} \frac{P_{i_{1} i_{2} ; j_{1} j_{2}}(h)}{h} \leq \frac{1}{1-3 \varepsilon} \liminf _{t \rightarrow 0} \frac{P_{i_{1} i_{2} ; j_{1} j_{2}}(t)}{t},
$$

and the required assertion follows immediately by letting $\varepsilon \rightarrow 0$. This completes the proof.

Set $\gamma(t)=\left(\gamma_{1}(t), \gamma_{2}(t)\right)$, then it is easy to see that almost every sample path of $\gamma(t)$ is a right continuous step function. Now letting $\mathbf{P}(t)=\left(P_{i_{1} i_{2} ; j_{1} j_{2}}(t)\right)_{N_{1} N_{2} \times N_{1} N_{2}}, \mathbf{Q}=$ $\left(q_{i_{1} i_{2} ; j_{1} j_{2}}\right)_{N_{1} N_{2} \times N_{1} N_{2}}=\mathbf{P}^{\prime}(0)$. Then by Chapman-Kolmogorov equation

$$
\mathbf{P}(t+h)=\mathbf{P}(t) \mathbf{P}(h)=\mathbf{P}(h) \mathbf{P}(t),
$$

we have

$$
\frac{\mathbf{P}(t+h)-\mathbf{P}(t)}{h}=\mathbf{P}(t)\left[\frac{\mathbf{P}(h)-\mathbf{I}}{h}\right]=\left[\frac{\mathbf{P}(h)-\mathbf{I}}{h}\right] \mathbf{P}(t) .
$$

Letting $h \rightarrow 0$ and taking limits give

$$
\begin{aligned}
\mathbf{P}^{\prime}(t) & =\mathbf{P}(t) \mathbf{Q}, \\
\mathbf{P}^{\prime}(t) & =\mathbf{Q P}(t) .
\end{aligned}
$$

Note that

$$
\mathbf{P}(0)=\mathbf{I} \text {. }
$$

Then by solving the ordinary differential equations (38) and (39), we obtain the following lemma.

Lemma 5. For $\mathbf{P}(t)$ and $\mathbf{Q}$ one has

$$
\mathbf{P}(t)=\exp \{\mathbf{Q} t\} .
$$

We are now in the position to give the sufficient conditions for the existence and uniqueness of the solution of (8). For this end, let us first give the definition of the solution. 
Definition 6. An $\mathbb{R}^{n}$-valued stochastic process $\{x(t)\}_{t_{0} \leq t \leq T}$ is called a solution of (8) if it has the following properties:

(i) $\{x(t)\}_{t_{0} \leq t \leq T}$ is continuous and $\mathscr{F}_{t}$-adapted;

(ii) $\left\{f\left(x(t), t, \gamma_{1}(t)\right)\right\}_{t_{0} \leq t \leq T} \in \mathscr{L}^{1}\left(\left[t_{0}, T\right] ; \mathbb{R}^{n}\right)$ while $\{g(x(t)$, $\left.\left.t, \gamma_{2}(t)\right)\right\}_{t_{0} \leq t \leq T} \in \mathscr{L}^{2}\left(\left[t_{0}, T\right] ; \mathbb{R}^{n \times m}\right) ;$

(iii) for any $t \in\left[t_{0}, T\right]$, equation

$$
\begin{aligned}
x(t)= & x\left(t_{0}\right)+\int_{t_{0}}^{t} f\left(x(s), s, \gamma_{1}(s)\right) d s \\
& +\int_{t_{0}}^{t} g\left(x(s), s, \gamma_{2}(s)\right) d B(s)
\end{aligned}
$$

holds with probability 1.

A solution $\{x(t)\}_{t_{0} \leq t \leq T}$ is said to be unique if any other solution $\{\tilde{x}(t)\}_{t_{0} \leq t \leq T}$ is indistinguishable from $\{x(t)\}_{t_{0} \leq t \leq T}$.

Now we can give our main results in this section.

Theorem 7. Assume that there exist two positive constants $\bar{K}$ and $K$ such that.

(Lipschitz condition) for all $x, y \in \mathbb{R}^{n}, t \in\left[t_{0}, T\right]$ and $\left(i_{1}, i_{2}\right) \in \mathbb{S}$

$$
\begin{aligned}
& \quad\left|f\left(x, t, i_{1}\right)-f\left(y, t, i_{1}\right)\right|^{2} \\
& \quad \vee\left|g\left(x, t, i_{2}\right)-g\left(y, t, i_{2}\right)\right|^{2} \leq \bar{K}|x-y|^{2} .
\end{aligned}
$$

(Linear growth condition) for all $\left(x, t,\left(i_{1}, i_{2}\right)\right) \in \mathbb{R}^{n} \times$ $\left[t_{0}, T\right] \times \mathbb{S}$

$$
\left|f\left(x, t, i_{1}\right)\right|^{2} \vee\left|g\left(x, t, i_{2}\right)\right|^{2} \leq K(1+|x|)^{2} .
$$

Then there exists a unique solution $x(t)$ to (8) and, moreover, the solution obeys

$$
\begin{aligned}
& E\left(\sup _{t_{0} \leq t \leq T}|x(t)|^{2}\right) \\
& \quad \leq\left(1+3 E\left|x_{0}\right|^{2}\right) \exp \left\{3 K\left(T-t_{0}\right)\left(T-t_{0}+4\right)\right\} .
\end{aligned}
$$

Proof. Recall that almost every sample path of $\gamma(t)=$ $\left(\gamma_{1}(t), \gamma_{2}(t)\right)$ is a right continuous step function with a finite number of jumps on $\left[t_{0}, T\right]$. Thus there exists a sequence of stopping times $\left\{\tau_{k}\right\}_{k \geq 0}$ such that

(i) for almost every $\omega \in \Omega$ there is a finite $\bar{k}_{\omega}$ for $t_{0}=$ $\tau_{0}<\tau_{1}<\cdots<\tau_{k}=T$ and $\tau_{k}=T$ if $k>\bar{k}_{\omega} ;$

(ii) both $\gamma_{1}(\cdot)$ and $\gamma_{2}(\cdot)$ in $\gamma(\cdot)$ are constants on interval $\left[\left[\tau_{k}, \tau_{k+1}[[\right.\right.$, namely,

$$
\begin{aligned}
& \gamma_{1}(t)=\gamma_{1}\left(\tau_{k}\right) \quad \text { on } \quad \tau_{k} \leq t \leq \tau_{k+1} \forall k \geq 0, \\
& \gamma_{2}(t)=\gamma_{2}\left(\tau_{k}\right) \quad \text { on } \quad \tau_{k} \leq t \leq \tau_{k+1} \quad \forall k \geq 0 .
\end{aligned}
$$

First of all, let us consider (8) on $t \in\left[\left[\tau_{0}, \tau_{1}[[\right.\right.$, then (8) becomes

$$
\begin{aligned}
d x(t)= & f\left(x(t), t, \gamma_{1}\left(t_{0}\right)\right) d t \\
& +g\left(x(t), t, \gamma_{2}\left(t_{0}\right)\right) d B(t)
\end{aligned}
$$

with initial conditions $x\left(t_{0}\right)=x_{0}, \gamma\left(t_{0}\right)=\left(\gamma_{1}\left(t_{0}\right), \gamma_{1}\left(t_{0}\right)\right)$. Then by the theory of SDEs, we obtain that (46) has a unique solution which obeys $x\left(\tau_{1}\right) \in L_{\mathscr{F}_{\tau_{1}}}^{2}\left(\Omega ; \mathbb{R}^{n}\right)$. We next consider (8) on $t \in\left[\left[\tau_{1}, \tau_{2}[[\right.\right.$ which becomes

$$
\begin{aligned}
d x(t)= & f\left(x(t), t, \gamma_{1}\left(\tau_{1}\right)\right) d t \\
& +g\left(x(t), t, \gamma_{2}\left(\tau_{1}\right)\right) d B(t) .
\end{aligned}
$$

Again by the theory of SDEs, (47) has a unique solution which obeys $x\left(\tau_{1}\right) \in L_{\mathscr{F}_{\tau_{2}}}^{2}\left(\Omega ; \mathbb{R}^{n}\right)$. Repeating this procedure, we conclude that (8) has a unique solution $x(t)$ on $\left[t_{0}, T\right]$.

Now, let us prove (44). For every $k \geq 1$, define the stopping time

$$
\tau_{k}=T \wedge \inf \left\{t \in\left[t_{0}, T\right]:|x(t)| \geq k\right\} .
$$

It is obvious that $\tau_{k} \uparrow T$ a.s. Set $x_{k}(t)=x\left(t \wedge \tau_{k}\right)$ for $t \in\left[t_{0}, T\right]$. Then $x_{k}(t)$ obeys the equation

$$
\begin{aligned}
x_{k}(t)= & x_{0}+\int_{t_{0}}^{t} f\left(x_{k}(s), s, \gamma_{1}(s)\right) I_{\left[\left[t_{0}, \tau_{k}\right]\right]}(s) d s \\
& +\int_{t_{0}}^{t} g\left(x_{k}(s), s, \gamma_{2}(s)\right) I_{\left[\left[t_{0}, \tau_{k}\right]\right]}(s) d B(s) .
\end{aligned}
$$

Making use of the elementary inequality $|a+b+c|^{2} \leq 3\left(|a|^{2}+\right.$ $|b|^{2}+|c|^{2}$ ), the Hölder inequality, and (43), we can see that

$$
\begin{aligned}
\left|x_{k}(t)\right|^{2}= & 3\left|x_{0}\right|^{2}+3 K\left(T-t_{0}\right) \int_{t_{0}}^{t}\left(1+\left|x_{k}(s)\right|^{2}\right) d s \\
& +3\left|\int_{t_{0}}^{t} g\left(x_{k}(s), s, \gamma_{2}(s)\right) I_{\left[\left[t_{0}, \tau_{k}\right]\right]}(s) d B(s)\right|^{2} .
\end{aligned}
$$

Thus, applying the Doob martingale inequality and (43), we can further show that

$$
\begin{aligned}
E\left(\sup _{t_{0} \leq s \leq t}\left|x_{k}(s)\right|^{2}\right) & \\
= & 3 E\left|x_{0}\right|^{2}+3 K\left(T-t_{0}\right) \int_{t_{0}}^{t}\left(1+E\left|x_{k}(s)\right|^{2}\right) d s \\
& +12 E \int_{t_{0}}^{t}\left|g\left(x_{k}(s), s, \gamma_{2}(s)\right)\right|^{2} I_{\left[\left[t_{0}, \tau_{k}\right]\right]}(s) d s \\
\leq & 3 E\left|x_{0}\right|^{2}+3 K\left(T-t_{0}+4\right) \int_{t_{0}}^{t}\left(1+E\left|x_{k}(s)\right|^{2}\right) d s .
\end{aligned}
$$

That is to say,

$$
\begin{aligned}
1+E\left(\sup _{t_{0} \leq s \leq t}\left|x_{k}(s)\right|^{2}\right) \leq & 1+3 E\left|x_{0}\right|^{2}+3 K\left(T-t_{0}+4\right) \\
& \times \int_{t_{0}}^{t}\left[1+E \sup _{t_{0} \leq r \leq s}\left|x_{k}(r)\right|^{2}\right] d s .
\end{aligned}
$$


Using the Gronwall inequality leads to

$$
\begin{aligned}
1+E & \left(\sup _{t_{0} \leq s \leq t}\left|x_{k}(s)\right|^{2}\right) \\
& \leq\left(1+3 E\left|x_{0}\right|^{2}\right) \exp \left\{3 K\left(T-t_{0}\right)\left(T-t_{0}+4\right)\right\} .
\end{aligned}
$$

Consequently,

$$
\begin{aligned}
1+E & \left(\sup _{t_{0} \leq t \leq \tau_{k}}|x(t)|^{2}\right) \\
& \leq\left(1+3 E\left|x_{0}\right|^{2}\right) \exp \left\{3 K\left(T-t_{0}\right)\left(T-t_{0}+4\right)\right\} .
\end{aligned}
$$

Then the required inequality (44) follows immediately by letting $k \rightarrow \infty$.

Condition (42) indicates that the coefficients $f\left(x, t, i_{1}\right)$ and $g\left(x, t, i_{2}\right)$ do not change faster than a linear function of $x$ as change in $x$. This means in particular the continuity of $f\left(x, t, i_{1}\right)$ and $g\left(x, t, i_{2}\right)$ in $x$ for all $t \in\left[t_{0}, T\right]$. Then functions that are discontinuous with respect to $x$ are excluded as the coefficients. Besides, there are many functions that do not satisfy the Lipschitz condition. These imply that the Lipschitz condition is too restrictive. To improve this Lipschitz condition let us introduce the concept of local solution.

Definition 8. Let $\sigma_{\infty}$ be a stopping time such that $t_{0} \leq \sigma_{\infty} \leq T$ a.s. An $\mathbb{R}^{n}$-valued $\mathscr{F}_{t}$-adapted continuous stochastic process $\{x(t)\}_{t_{0} \leq t<\sigma_{\infty}}$ is called a local solution of (8) if $x\left(t_{0}\right)=x_{0}$ and, moreover, there is a nondecreasing sequence $\left\{\sigma_{k}\right\}_{k \geq 1}$ such that $t_{0} \leq\left\{\sigma_{k}\right\} \uparrow \sigma_{\infty}$ a.s. and

$$
\begin{aligned}
x(t)= & x\left(t_{0}\right)+\int_{t_{0}}^{t \wedge \sigma_{k}} f\left(x(s), s, \gamma_{1}(s)\right) d s \\
& +\int_{t_{0}}^{t \wedge \sigma_{k}} g\left(x(s), s, \gamma_{2}(s)\right) d B(s)
\end{aligned}
$$

holds for any $t \in\left[t_{0}, T\right)$ and $k \geq 1$ with probability one. If, furthermore,

$$
\limsup _{t \rightarrow+\sigma_{\infty}}|x(t)|=\infty \quad \text { whenever } \sigma_{\infty}<T,
$$

then it is called a maximal local solution and $\sigma_{\infty}$ is called the explosion time. A maximal local solution $\left\{x(t): t_{0} \leq t<\sigma_{\infty}\right\}$ is said to be unique if any other maximal local solution $\{\bar{x}(t)$ : $\left.t_{0} \leq t<\bar{\sigma}_{\infty}\right\}$ is indistinguishable from it, namely, $\sigma_{\infty}=\bar{\sigma}_{\infty}$ and $x(t)=\bar{x}(t)$ for $t_{0} \leq t<\sigma_{\infty}$ with probability one.

Definition 9 (local Lipschitz condition). For every integer $k \geq 1$, there exists a positive constant $h_{k}$ such that, for all $t \in\left[t_{0}, T\right], i=\left(i_{1}, i_{2}\right) \in \mathbb{S}$ and those $x, y \in \mathbb{R}^{n}$ with $|x| \vee|y| \leq k$

$$
\begin{aligned}
& \left|f\left(x, t, i_{1}\right)-f\left(y, t, i_{1}\right)\right|^{2} \\
& \quad \vee\left|g\left(x, t, i_{2}\right)-g\left(y, t, i_{2}\right)\right|^{2} \leq h_{k}|x-y|^{2} .
\end{aligned}
$$

The following theorem shows the existence of unique maximal local solution under the local Lipschitz condition without the linear growth condition.
Theorem 10. Under condition (57), there exists a unique maximal local solution of (8).

Proof. Define functions

$$
\begin{aligned}
& f_{k}\left(x, t, i_{1}\right)= \begin{cases}f\left(x, t, i_{1}\right), & \text { if }|x| \leq k, \\
f\left(\frac{k x}{|x|}, t, i_{1}\right), & \text { if }|x|>k,\end{cases} \\
& g_{k}\left(x, t, i_{2}\right)= \begin{cases}g\left(x, t, i_{2}\right), & \text { if }|x| \leq k, \\
g\left(\frac{k x}{|x|}, t, i_{2}\right), & \text { if }|x|>k .\end{cases}
\end{aligned}
$$

Then $f_{k}$ and $g_{k}$ satisfy the Lipschitz condition and the linear growth condition. Thus by Theorem 7 , there is a unique solution $x_{k}(t)$ of the equation

$$
\begin{aligned}
d x_{k}(t)= & f_{k}\left(x_{k}(t), t, \gamma_{1}(t)\right) d t \\
& +g_{k}\left(x_{k}(t), t, \gamma_{2}(t)\right) d B(t)
\end{aligned}
$$

with the initial conditions $x_{k}\left(t_{0}\right)=x_{0}$ and $\gamma\left(t_{0}\right)=\left(\gamma_{1}\left(t_{0}\right)\right.$, $\left.\gamma_{2}\left(t_{0}\right)\right)$. Define the stopping times

$$
\sigma_{k}=T \wedge \inf \left\{t \in\left[t_{0}, T\right]:\left|x_{k}(t)\right| \geq k\right\} .
$$

Clearly, if $t_{0} \leq t \leq \sigma_{k}$,

$$
x_{k}(t)=x_{k+1}(t),
$$

which indicates that $\sigma_{k}$ is increasing so $\sigma_{k}$ has its limit $\sigma_{\infty}=$ $\lim _{k \rightarrow+\infty} \sigma_{k}$. Define $\left\{x(t): t_{0} \leq t<\sigma_{\infty}\right\}$ by

$$
x(t)=x_{k}(t), \quad t \in\left[\left[\sigma_{k-1}, \sigma_{k}[[, k \geq 1,\right.\right.
$$

where $\sigma_{0}=t_{0}$. Applying (61), one can show that $x\left(t \wedge \sigma_{k}\right)=$ $x_{k}\left(t \wedge \sigma_{k}\right)$. It then follows from (59) that

$$
\begin{aligned}
x\left(t \wedge \sigma_{k}\right)= & x_{0}+\int_{t_{0}}^{t \wedge \sigma_{k}} f_{k}\left(x(s), s, \gamma_{1}(s)\right) d s \\
& +\int_{t_{0}}^{t \wedge \sigma_{k}} g_{k}\left(x(s), s, \gamma_{2}(s)\right) d B(s) \\
= & x_{0}+\int_{t_{0}}^{t \wedge \sigma_{k}} f\left(x(s), s, \gamma_{1}(s)\right) d s \\
& +\int_{t_{0}}^{t \wedge \sigma_{k}} g\left(x(s), s, \gamma_{2}(s)\right) d B(s),
\end{aligned}
$$

for any $t \in\left[t_{0}, T\right)$ and $k \geq 1$. It is easy to see that if $\sigma_{\infty} \leq T$, then

$$
\begin{aligned}
& \limsup _{t \rightarrow \sigma_{\infty}}|x(t)| \\
& \quad \geq \limsup _{k \rightarrow+\infty}\left|x\left(\sigma_{k}\right)\right|=\limsup _{k \rightarrow+\infty}\left|x_{k}\left(\sigma_{k}\right)\right|=\infty .
\end{aligned}
$$

Therefore $\left\{x(t): t_{0} \leq t<\sigma_{\infty}\right\}$ is a maximal local solution.

Now, we will prove the uniqueness. Let $\left\{\bar{x}(t): t_{0} \leq t<\right.$ $\left.\bar{\sigma}_{\infty}\right\}$ be another maximal local solution. Define

$$
\bar{\sigma}_{k}=\bar{\sigma}_{\infty} \wedge \inf \left\{t \in \left[\left[t_{0}, \bar{\sigma}_{\infty}[[: \bar{x}(t) \geq k\} .\right.\right.\right.
$$


Then $\bar{\sigma}_{k} \rightarrow \bar{\sigma}_{\infty}$ a.s. and

$$
P\left\{x(t)=\bar{x}(t), t \in\left[\left[t_{0}, \sigma_{k} \wedge \bar{\sigma}_{k}\right]\right]\right\}=1 \quad \forall k \geq 1
$$

Letting $k \rightarrow \infty$ gives

$$
P\left\{x(t)=\bar{x}(t), t \in\left[\left[t_{0}, \sigma_{\infty} \wedge \bar{\sigma}_{\infty}[[\}=1\right.\right.\right.
$$

In order to complete the proof, we need only to show that $\sigma_{\infty}=\bar{\sigma}_{\infty}$ a.s. In fact, for almost every $\omega \in\left\{\sigma_{\infty}<\bar{\sigma}_{\infty}\right\}$, we have

$$
\begin{aligned}
& \left|\bar{x}\left(\sigma_{\infty}, \omega\right)\right| \\
& \quad=\lim _{k \rightarrow+\infty}\left|\bar{x}\left(\sigma_{k}, \omega\right)\right|=\lim _{k \rightarrow+\infty}\left|x\left(\sigma_{k}, \omega\right)\right|=\infty,
\end{aligned}
$$

which contradicts the fact that $\bar{x}(t, \omega)$ is continuous on $t \epsilon$ $\left[t_{0}, \bar{\sigma}_{\infty}(\omega)\right)$. This implies $\sigma_{\infty} \geq \bar{\sigma}_{\infty}$ a.s. In the same way, one can show $\sigma_{\infty} \leq \bar{\sigma}_{\infty}$ a.s. Thus we must have $\sigma_{\infty}=\bar{\sigma}_{\infty}$ a.s. This completes the proof.

In many situations, we often consider an SDE on $\left[t_{0}, \infty\right)$

$$
\begin{aligned}
d x(t)= & f\left(x(t), t, \gamma_{1}(t)\right) d t \\
& +g\left(x(t), t, \gamma_{2}(t)\right) d B(t), \quad t_{0} \leq t<\infty
\end{aligned}
$$

with initial data $x\left(t_{0}\right)=x_{0}$ and $\gamma\left(t_{0}\right)$. If the assumption of the existence-and-uniqueness theorem holds on every finite subinterval $\left[t_{0}, T\right]$ of $\left[t_{0}, \infty\right)$, then (69) has a unique solution $x(t)$ on the entire interval $\left[t_{0}, \infty\right)$. Such a solution is called a global solution. To establish a more general result about global solution, we need more notations. To this end, we introduce an operator $L V$ from $\mathbb{R}^{n} \times \mathbb{R}_{+} \times \mathbb{S}$ to $\mathbb{R}$ which is given by

$$
\begin{aligned}
L V\left(x, t,\left(i_{1}, i_{2}\right)\right) \\
=V_{t}\left(x, t,\left(i_{1}, i_{2}\right)\right)+V_{x}\left(x, t,\left(i_{1}, i_{2}\right)\right) f\left(x, t, i_{1}\right) \\
\quad+0.5 \operatorname{trace}\left[g^{T}\left(x, t, i_{2}\right) V_{x x}\left(x, t,\left(i_{1}, i_{2}\right)\right) g\left(x, t, i_{2}\right)\right] \\
\quad+\sum_{\left(j_{1}, j_{2}\right) \in \mathbb{S}} q_{i_{1} i_{2} ; j_{1} j_{2}} V\left(x, t,\left(j_{1}, j_{2}\right)\right),
\end{aligned}
$$

where $V\left(x, t,\left(i_{1}, i_{2}\right)\right) \in C^{2,1}\left(\mathbb{R}^{n} \times \mathbb{R}_{+} \times \mathbb{S} ; \mathbb{R}_{+}\right)$and

$$
\begin{gathered}
V_{t}\left(x, t,\left(i_{1}, i_{2}\right)\right)=\frac{\partial V\left(x, t,\left(i_{1}, i_{2}\right)\right)}{\partial t} ; \\
V_{x}\left(x, t,\left(i_{1}, i_{2}\right)\right) \\
=\left(\frac{\partial V\left(x, t,\left(i_{1}, i_{2}\right)\right)}{\partial x_{1}}, \ldots, \frac{\partial V\left(x, t,\left(i_{1}, i_{2}\right)\right)}{\partial x_{n}}\right) ; \\
V_{x x}\left(x, t,\left(i_{1}, i_{2}\right)\right)=\left(\frac{\partial^{2} V\left(x, t,\left(i_{1}, i_{2}\right)\right)}{\partial x_{i} \partial x_{j}}\right)_{n \times n} .
\end{gathered}
$$

Theorem 11. Assume that the local Lipschitz condition (57) holds. Assume also that there is a function $V\left(x, t,\left(i_{1}, i_{2}\right)\right) \in$ $C^{2,1}\left(\mathbb{R}^{n} \times \mathbb{R}_{+} \times \mathbb{S} ; \mathbb{R}_{+}\right)$and a constant $\theta>0$ such that

$$
\begin{array}{r}
\lim _{|x| \rightarrow \infty}\left(\inf _{\left(t,\left(i_{1}, i_{2}\right)\right) \in \mathbb{R}_{+} \times \mathbb{S}} V\left(x, t,\left(i_{1}, i_{2}\right)\right)\right)=\infty, \\
\operatorname{LV}\left(x, t,\left(i_{1}, i_{2}\right)\right) \leq \theta\left(1+V\left(x, t,\left(i_{1}, i_{2}\right)\right)\right), \\
\forall\left(x, t,\left(i_{1}, i_{2}\right)\right) \in \mathbb{R}^{n} \times \mathbb{R}_{+} \times \mathbb{S} .
\end{array}
$$

Then there exists a unique global solution $x(t)$ to (69).

Proof. We need only to prove the theorem for any initial condition $x_{0} \in \mathbb{R}^{n}$ and $\left(\gamma_{1}\left(t_{0}\right), \gamma_{2}\left(t_{0}\right)\right) \in \mathbb{S}$. From Theorem 10, we know that the local Lipschitz condition guarantees the existence of the unique maximal solution $x(t)$ on $\left[t_{0}, \sigma_{\infty}\right)$, where $\sigma_{\infty}$ is the explosion time. We need only to show $\sigma_{\infty}=$ $\infty$ a.s. If this is not true, then we can find a pair of positive constants $\varepsilon$ and $T$ such that

$$
P\left\{\sigma_{\infty} \leq T\right\}>2 \varepsilon
$$

For each integer $k \geq 1$, define the stopping time

$$
\sigma_{k}=\inf \left\{t \geq t_{0}:|x(t)| \geq k\right\} .
$$

Since $\sigma_{k} \rightarrow \sigma_{\infty}$ almost surely, we can find a sufficiently large integer $k_{0}$ for

$$
P\left\{\sigma_{k} \leq T\right\}>\varepsilon, \quad \forall k \geq k_{0} .
$$

Fix any $k \geq k_{0}$, then for any $t_{0} \leq t \leq T$, by virtue of the generalized Itô formula (see, e.g., [1])

$$
\begin{aligned}
E V(x & \left.\left(t \wedge \sigma_{k}\right), t \wedge \sigma_{k}, \gamma\left(t \wedge \sigma_{k}\right)\right) \\
= & V\left(x_{0}, t_{0}, \gamma_{0}\right)+E \int_{t_{0}}^{t \wedge \sigma_{k}} L V(x(s), s, \gamma(s)) d s \\
\leq & V\left(x_{0}, t_{0}, \gamma_{0}\right)+\theta\left(T-t_{0}\right) \\
& +\theta \int_{t_{0}}^{t} E V\left(x\left(s \wedge \sigma_{k}\right), s \wedge \sigma_{k}, \gamma\left(s \wedge \sigma_{k}\right)\right) d s .
\end{aligned}
$$

Making use of the Gronwall inequality gives

$$
\begin{aligned}
E V( & \left.x\left(t \wedge \sigma_{k}\right), t \wedge \sigma_{k}, \gamma\left(t \wedge \sigma_{k}\right)\right) \\
& \leq\left[V\left(x_{0}, t_{0}, \gamma_{0}\right)+\theta\left(T-t_{0}\right)\right] \exp \left\{\theta\left(T-t_{0}\right)\right\} .
\end{aligned}
$$

Therefore

$$
\begin{aligned}
& E\left(I_{\left\{\sigma_{k} \leq T\right\}} V\left(x\left(\sigma_{k}\right), \sigma_{k}, \gamma\left(\sigma_{k}\right)\right)\right) \\
& \quad \leq\left[V\left(x_{0}, t_{0}, \gamma_{0}\right)+\theta\left(T-t_{0}\right)\right] \exp \left\{\theta\left(T-t_{0}\right)\right\} .
\end{aligned}
$$

At the same time, set

$$
g_{k}=\inf \left\{V\left(x, t,\left(i_{1}, i_{2}\right)\right):|x| \geq k, t \in\left[t_{0}, T\right],\left(i_{1}, i_{2}\right) \in \mathbb{S}\right\} .
$$


Then (72) means $g_{k} \rightarrow \infty$. It follows from (76) and (79) that

$$
\begin{gathered}
{\left[V\left(x_{0}, t_{0}, \gamma_{0}\right)+\theta\left(T-t_{0}\right)\right] \exp \left\{\theta\left(T-t_{0}\right)\right\}} \\
\geq g_{k} P\left\{\sigma_{k} \leq T\right\} \geq \varepsilon g_{k} .
\end{gathered}
$$

Letting $k \rightarrow \infty$ yields a contradiction, that is to say, $\sigma_{\infty}=\infty$. The proof is complete.

\section{3. $L^{P}$-Estimates}

In the previous section, we have investigated the existence and uniqueness of the solution to (8). In this section, as above, let $x(t), t_{0} \leq t \leq T$ be the unique solution of (8) with initial conditions $x\left(t_{0}\right)=x_{0}$ and $\gamma\left(t_{0}\right)$, and we will estimate the $p$ th moment of the solution.

Theorem 12. Assume that there is a function $V\left(x, t,\left(i_{1}, i_{2}\right)\right) \in$ $C^{2,1}\left(\mathbb{R}^{n} \times \mathbb{R}_{+} \times \mathbb{S} ; \mathbb{R}_{+}\right)$and positive constants $p, \eta, \theta$ such that for all $\left(x, t,\left(i_{1}, i_{2}\right)\right) \in \mathbb{R}^{n} \times \mathbb{R}_{+} \times \mathbb{S}$,

$$
\begin{gathered}
\eta|x|^{p} \leq V\left(x, t,\left(i_{1}, i_{2}\right)\right), \\
L V\left(x, t,\left(i_{1}, i_{2}\right)\right) \leq \theta V\left(x, t,\left(i_{1}, i_{2}\right)\right) .
\end{gathered}
$$

Assume also the initial condition $x\left(t_{0}\right)=x_{0}$ and $\gamma\left(t_{0}\right)$ obeys $E V\left(x_{0}, t_{0}, \gamma\left(t_{0}\right)\right)<\infty$, then one has

$$
\begin{aligned}
& E|x(t)|^{p} \\
& \quad \leq \frac{E V\left(x_{0}, t_{0}, \gamma\left(t_{0}\right)\right) \exp \left\{\theta\left(t-t_{0}\right)\right\}}{\eta}, \quad \forall t \in\left[t_{0}, T\right] .
\end{aligned}
$$

Proof. For each integer $k \geq 1$, define the stopping time

$$
\sigma_{k}=T \wedge \inf \left\{t \geq t_{0}:|x(t)| \geq k\right\} .
$$

Thus $\sigma_{k} \rightarrow T$ a.s. Using the generalized Itô's formula and (83), we obtain that for $t \in\left[t_{0}, T\right]$

$$
\begin{aligned}
& E V(x(\left.\left.t \wedge \sigma_{k}\right), t \wedge \sigma_{k}, \gamma\left(t \wedge \sigma_{k}\right)\right) \\
& \leq E V\left(x_{0}, t_{0}, \gamma\left(t_{0}\right)\right) \\
& \quad+\theta \int_{t_{0}}^{t} E V\left(x\left(s \wedge \sigma_{k}\right), s \wedge \sigma_{k}, \gamma\left(s \wedge \sigma_{k}\right)\right) d s .
\end{aligned}
$$

Then the Gronwall inequality indicates

$$
\begin{aligned}
& E V\left(x\left(t \wedge \sigma_{k}\right), t \wedge \sigma_{k}, \gamma\left(t \wedge \sigma_{k}\right)\right) \\
& \quad \leq E V\left(x_{0}, t_{0}, \gamma\left(t_{0}\right)\right) \exp \left\{\theta\left(t-t_{0}\right)\right\}
\end{aligned}
$$

for all $t \in\left[t_{0}, T\right]$. By virtue of condition (83) we obtain the required assertion (84).

Corollary 13. Assume $p \geq 2$ and $x_{0} \in L_{\mathscr{F}_{t_{0}}}^{p}\left(\Omega ; \mathbb{R}^{n}\right)$. Assume also that there exists a constant $\theta>0$ such that, for all $\left(x, t,\left(i_{1}, i_{2}\right)\right) \in \mathbb{R}^{n} \times \mathbb{R}_{+} \times \mathbb{S}$,

$$
x^{T} f\left(x, t, i_{1}\right)+\frac{p-1}{2}\left|g\left(x, t, i_{2}\right)\right|^{2} \leq \theta\left(1+|x|^{2}\right) .
$$

Then one has

$$
\begin{aligned}
& E|x(t)|^{p} \\
& \quad \leq 2^{(p-2) / 2}\left(1+E\left|x_{0}\right|^{p}\right) \exp \left\{p \theta\left(t-t_{0}\right)\right\} \quad \forall t \in\left[t_{0}, T\right] .
\end{aligned}
$$

Proof. Define $V\left(x, t,\left(i_{1}, i_{2}\right)\right)=\left(1+|x|^{2}\right)^{p / 2}$. Making use of (88) yields

$$
\begin{aligned}
L V & \left(x, t,\left(i_{1}, i_{2}\right)\right) \\
= & p\left(1+|x|^{2}\right)^{(p-2) / 2} x^{T} f\left(x, t, i_{1}\right) \\
& +\frac{p}{2}\left(1+|x|^{2}\right)^{(p-2) / 2}\left|g\left(x, t, i_{2}\right)\right|^{2} \\
& +\frac{p(p-2)}{2}\left(1+|x|^{2}\right)^{(p-4) / 2}\left|x^{T} g\left(x, t, i_{2}\right)\right|^{2} \\
\leq & p\left(1+|x|^{2}\right)^{(p-2) / 2}\left[x^{T} f\left(x, t, i_{1}\right)+\frac{p-1}{2}\left|g\left(x, t, i_{2}\right)\right|^{2}\right] \\
\leq & p \theta\left(1+|x|^{2}\right)^{p / 2} .
\end{aligned}
$$

Then by Theorem 12, we get

$$
\begin{aligned}
E(1+ & \left.|x(t)|^{2}\right)^{p / 2} \\
& \leq E\left(1+\left|x_{0}\right|^{2}\right)^{p / 2} \exp \left\{p \theta\left(t-t_{0}\right)\right\}
\end{aligned}
$$

and the required assertion (89) follows.

It is useful to point out that if the linear growth condition (43) is satisfied, then (88) is fulfilled with $\theta=\sqrt{K}+K(p-$ $1) / 2$. Now, we will show the other important properties of the solution.

Theorem 14. Let $p \geq 2$ and $x_{0} \in L_{\mathscr{F}_{t_{0}}}^{p}\left(\Omega ; \mathbb{R}^{n}\right)$. Assume also that the linear growth condition (43) holds. Then one has

$$
E|x(t)-x(s)|^{p} \leq C(t-s)^{p / 2},
$$

where

$$
\begin{aligned}
C= & 2^{p-2}\left(1+E\left|x_{0}\right|^{p}\right) \exp \left\{p \theta\left(T-t_{0}\right)\right\} \\
& \times\left(\left[2\left(T-t_{0}\right)\right]^{p / 2}+[p(p-1)]^{p / 2}\right)
\end{aligned}
$$

and $\theta=\sqrt{K}+K(p-1) / 2$. Particularly, the pth moment of the solution is continuous on $\left[t_{0}, T\right]$. 
Proof. Applying the elementary inequality $|a+b|^{p} \leq$ $2^{p-1}\left(|a|^{p}+|b|^{p}\right)$, the Hölder inequality, and the linear growth condition, we can derive that

$$
\begin{aligned}
E \mid x( & (t)-\left.x(s)\right|^{p} \\
\leq & 2^{p-1} E\left|\int_{s}^{t} f\left(x(u), u, \gamma_{1}(u)\right) d u\right|^{p} \\
& +2^{p-1} E\left|\int_{s}^{t} g\left(x(u), u, \gamma_{2}(u)\right) d B(u)\right|^{p} \\
\leq & {[2(t-s)]^{p-1} E \int_{s}^{t}\left|f\left(x(u), u, \gamma_{1}(u)\right)\right|^{p} d u } \\
& +0.5[2 p(p-1)]^{p / 2}(t-s)^{(p-2) / 2} E \\
& \times \int_{s}^{t}\left|g\left(x(u), u, \gamma_{2}(u)\right)\right|^{p} d u \\
\leq & C_{1}(t-s)^{(p-2) / 2} \int_{s}^{t} E\left(1+|x(u)|^{2}\right)^{p / 2} d u,
\end{aligned}
$$

where $C_{1}=2^{(p-2) / 2} K^{p / 2}\left(\left[2\left(T-t_{0}\right)\right]^{p / 2}+[p(p-1)]^{p / 2}\right)$. Using (91) yields

$$
\begin{aligned}
E \mid x( & t)-\left.x(s)\right|^{p} \\
\leq & C_{1}(t-s)^{(p-2) / 2} \\
& \times \int_{s}^{t} 2^{(p-2) / 2}\left(1+E\left|x_{0}\right|^{p}\right) \exp \left\{p \theta\left(u-t_{0}\right)\right\} d u \\
\leq & C_{1} 2^{(p-2) / 2}\left(1+E\left|x_{0}\right|^{p}\right) \\
& \times \exp \left\{p \theta\left(T-t_{0}\right)\right\}(t-s)^{(p / 2)},
\end{aligned}
$$

which is the desired inequality.

Theorem 15. Let $p \geq 2$ and $x_{0} \in L_{\mathscr{F}_{t_{0}}}^{p}\left(\Omega ; \mathbb{R}^{n}\right)$. Assume that there is a $K>0$ such that for all $\left(x, t,\left(i_{1}, i_{2}\right)\right) \in \mathbb{R}^{n} \times\left[t_{0}, T\right] \times \mathbb{S}$

$$
x^{T}\left|f\left(x, t, i_{1}\right)\right| \vee\left|g\left(x, t, i_{2}\right)\right|^{2} \leq K(1+|x|)^{2} .
$$

Then

$$
\begin{aligned}
& E\left(\sup _{t_{0} \leq t \leq T}|x(t)|^{p}\right) \\
& \quad \leq\left(1+2 E\left|x_{0}\right|^{p}\right) \exp \left\{2 p(10 p+1) K\left(T-t_{0}\right)\right\} .
\end{aligned}
$$

Proof. Making use of the generalized Itô's formula and condition (96), we have

$$
\begin{aligned}
& |x(t)|^{p} \\
& \leq\left|x_{0}\right|^{p}+M(t) \\
& +\int_{t_{0}}^{t} p|x(s)|^{p-2}\left[x^{T}(s) f\left(x(s), s, \gamma_{1}(s)\right)\right. \\
& \left.+\frac{p-1}{2}\left|g\left(x(s), s, \gamma_{2}(s)\right)\right|^{2}\right] d s \\
& \leq\left|x_{0}\right|^{p}+M(t)+0.5 p(p+1) K \\
& \times \int_{t_{0}}^{t}|x(s)|^{p-2}\left(1+|x(s)|^{2}\right) d s \\
& \leq\left|x_{0}\right|^{p}+M(t)+p(p+1) K \int_{t_{0}}^{t}\left(1+|x(s)|^{p}\right) d s,
\end{aligned}
$$

where

$$
M(t)=\int_{t_{0}}^{t} p|x(s)|^{p-2} x^{T}(s) g\left(x(s), s, \gamma_{2}(s)\right) d B(s) .
$$

Therefore, for any $t_{1} \in\left[t_{0}, T\right]$,

$$
\begin{aligned}
E\left(\sup _{t_{0} \leq t \leq t_{1}}|x(t)|^{p}\right) \leq & E\left|x_{0}\right|^{p}+E\left(\sup _{t_{0} \leq t \leq t_{1}}|M(t)|\right) \\
& +p(p+1) K E \int_{t_{0}}^{t_{1}}\left(1+|x(s)|^{p}\right) d s .
\end{aligned}
$$

At the same time, applying the well-known BurkholderDavis-Gundy inequality (see, e.g., [2]) gives

$$
\begin{aligned}
E\left(\sup _{t_{0} \leq t \leq t_{1}}|M(t)|\right) & \\
\leq & 3 E\left(\int_{t_{0}}^{t_{1}} p^{2}|x(s)|^{2 p-2}\left|g\left(x(s), s, \gamma_{2}(s)\right)\right|^{2} d s\right)^{0.5} \\
\leq & 3 E\left(\sup _{t_{0} \leq t \leq t_{1}}|x(t)|^{p}\right. \\
\quad & \left.\quad \int_{t_{0}}^{t_{1}} p^{2}|x(s)|^{p-2}\left|g\left(x(s), s, \gamma_{2}(s)\right)\right|^{2} d s\right)^{0.5} \\
\leq & 0.5 E\left(\sup _{t_{0} \leq t \leq t_{1}}|x(t)|^{p}\right)+4.5 E \\
& \times \int_{t_{0}}^{t_{1}} p^{2} K|x(s)|^{p-2}\left(1+|x(s)|^{2}\right) d s \\
\leq & 0.5 E\left(\sup _{t_{0} \leq t \leq t_{1}}|x(t)|^{p}\right)+9 p^{2} K E \int_{t_{0}}^{t_{1}}\left(1+|x(s)|^{p}\right) d s .
\end{aligned}
$$


Substituting the above inequality into (100) gives

$$
\begin{aligned}
E\left(\sup _{t_{0} \leq t \leq t_{1}}|x(t)|^{p}\right) \leq & 2 E\left|x_{0}\right|^{p} \\
& +2 p(10 p+1) K \int_{t_{0}}^{t_{1}}\left(1+|x(s)|^{p}\right) d s .
\end{aligned}
$$

Thus

$$
\begin{aligned}
1+E & \left(\sup _{t_{0} \leq t \leq t_{1}}|x(t)|^{p}\right) \\
\leq & 1+2 E\left|x_{0}\right|^{p}+2 p(10 p+1) K \\
& \times \int_{t_{0}}^{t_{1}}\left(1+\sup _{t_{0} \leq t \leq s}|x(t)|^{p}\right) d s .
\end{aligned}
$$

Then the required assertion follows from the Gronwall inequality.

Up to now, we have discussed the $L^{p}$-estimates for the solution in the case when $p \geq 2$. As for $0<p<2$, the similar results can be given without any difficulty as long as we note that the Hölder inequality implies

$$
E|x(t)|^{p} \leq\left[E|x(t)|^{2}\right]^{0.5 p} \text {. }
$$

\section{Example}

Consider the following Black-Scholes model:

$$
d X(t)=\mu\left(\gamma_{1}(t)\right) X(t) d t+\nu\left(\gamma_{2}(t)\right) X(t) d B(t)
$$

where $\gamma_{1}(t)$ is a right-continuous homogenous Markovian chain taking values in finite state spaces $\mathbb{S}_{1}=\{1,2\}$ and $\gamma_{2}(t)$ is a right-continuous homogenous Markovian chain taking values in finite state spaces $\mathbb{S}_{2}=\{1,2,3\}, \mu(i)=i, i=1,2$, $v(j)=j+1, j=1,2,3$. Taking $\bar{K}=16, K=9$, then (42) and (43) hold. Therefore, by Theorem 7, (105) has a unique solution.

\section{Conclusions and Further Research}

This paper is devoted to studying the existence and uniqueness of solution of SDEs with multi-Markovian switchings and estimating the $p$ th moment of the solution. We have used two continuous-time Markovian chains to model the SDEs. This area is becoming increasingly useful in engineering, economics, communication theory, active networking, and so forth. The sufficient criteria for existence and uniqueness of solution, local solution, and maximal local solution were established. Those results indicate that (8) keeps many properties that (89) owns. At the same time, although the hypothesis (H1) is used in this paper, we want to point out that this hypothesis is not essential. In fact, (H1) can be replaced by the following generalized hypothesis.
$(\mathrm{H} 1)^{\prime}$ : both $\gamma_{1}(t)$ and $\gamma_{2}(t)$ are right-continuous homogenous Markovian chains such that $\gamma(t)=\left(\gamma_{1}(t), \gamma_{2}(t)\right)$ is a homogenous vector chain.

Under hypothesis $(\mathrm{H} 1)^{\prime}$, the results given in this paper can be established similarly. It is easy to see that if $\gamma_{1}(t) \equiv$ $\gamma_{2}(t)$ and $\gamma_{1}(t)$ is a right-continuous homogenous Markovian chain, then $(\mathrm{H} 1)^{\prime}$ is fulfilled immediately. At the same time, if $\gamma_{1}(t) \equiv \gamma_{2}(t)$, (8) will reduce to the classical SDEs with single Markovian chain; that is to say, the classical theory about SDEs with single Markovian chain is a special case of our theory. On the other hand, many theorems in this paper will play important roles in further study. For example, Theorem 15 will be useful when one studies the approximate solutions.

Some important and interesting questions can be further investigated using the results in this paper. For example, approximate solutions, boundedness and stability, stochastic functional differential equations with vector Markovian switching and their applications. In particular, the stability of (8) is one of the most important and interesting topics, and those investigations are in progress.

\section{Acknowledgments}

The authors thank the editor and referees for their very important and helpful comments and suggestions. The authors also thank Dr. C. Zhang for helping them to improve the English exposition. This research is supported by the NSFC of China (nos. 11171081 and 11171056).

\section{References}

[1] A. V. Skorokhod, Asymptotic Methods in the Theory of Stochastic Differential Equations, vol. 78 of Translations of Mathematical Monographs, American Mathematical Society, Providence, RI, USA, 1989.

[2] X. Mao and C. Yuan, Stochastic Differential Euations with Markovian Switching, Imperial College Press, London, UK, 2006.

[3] G. Yin and X. Y. Zhou, "Markowitz's mean-variance portfolio selection with regime switching: from discrete-time models to their continuous-time limits," IEEE Transactions on Automatic Control, vol. 49, no. 3, pp. 349-360, 2004.

[4] J. Buffington and R. J. Elliott, "American options with regime switching," International Journal of Theoretical and Applied Finance, vol. 5, no. 5, pp. 497-514, 2002.

[5] C. Zhu and G. Yin, "On competitive Lotka-Volterra model in random environments," Journal of Mathematical Analysis and Applications, vol. 357, no. 1, pp. 154-170, 2009.

[6] Q. Luo and X. Mao, "Stochastic population dynamics under regime switching," Journal of Mathematical Analysis and Applications, vol. 334, no. 1, pp. 69-84, 2007.

[7] X. Li, D. Jiang, and X. Mao, "Population dynamical behavior of Lotka-Volterra system under regime switching," Journal of Computational and Applied Mathematics, vol. 232, no. 2, pp. 427-448, 2009.

[8] X. Li, A. Gray, D. Jiang, and X. Mao, "Sufficient and necessary conditions of stochastic permanence and extinction for stochastic logistic populations under regime switching," Journal 
of Mathematical Analysis and Applications, vol. 376, no. 1, pp. 1128, 2011.

[9] M. Liu and K. Wang, "Asymptotic properties and simulations of a stochastic logistic model under regime switching," Mathematical and Computer Modelling, vol. 54, no. 9-10, pp. 2139-2154, 2011.

[10] G. Hu and K. Wang, "Stability in distribution of competitive Lotka-Volterra system with Markovian switching," Applied Mathematical Modelling, vol. 35, no. 7, pp. 3189-3200, 2011.

[11] M. Liu and K. Wang, "Asymptotic properties and simulations of a stochastic logistic model under regime switching II," Mathematical and Computer Modelling, vol. 55, no. 3-4, pp. 405418, 2012.

[12] Z. Wu, H. Huang, and L. Wang, "Stochastic delay logistic model under regime switching," Abstract and Applied Analysis, vol. 2012, Article ID 241702, 26 pages, 2012. 


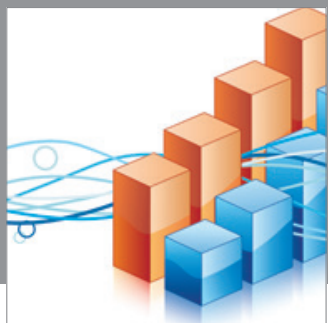

Advances in

Operations Research

mansans

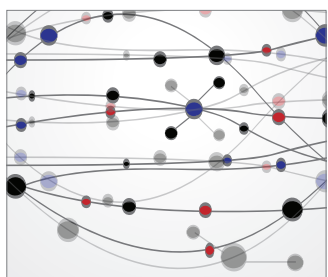

The Scientific World Journal
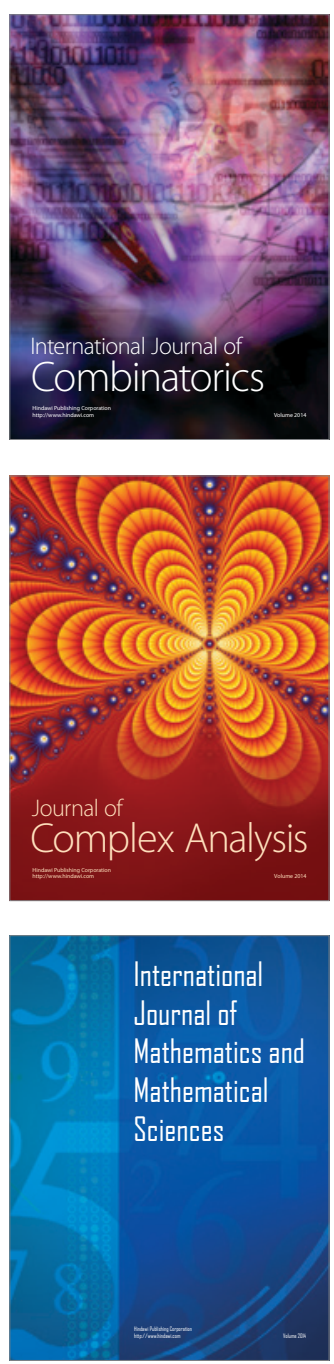
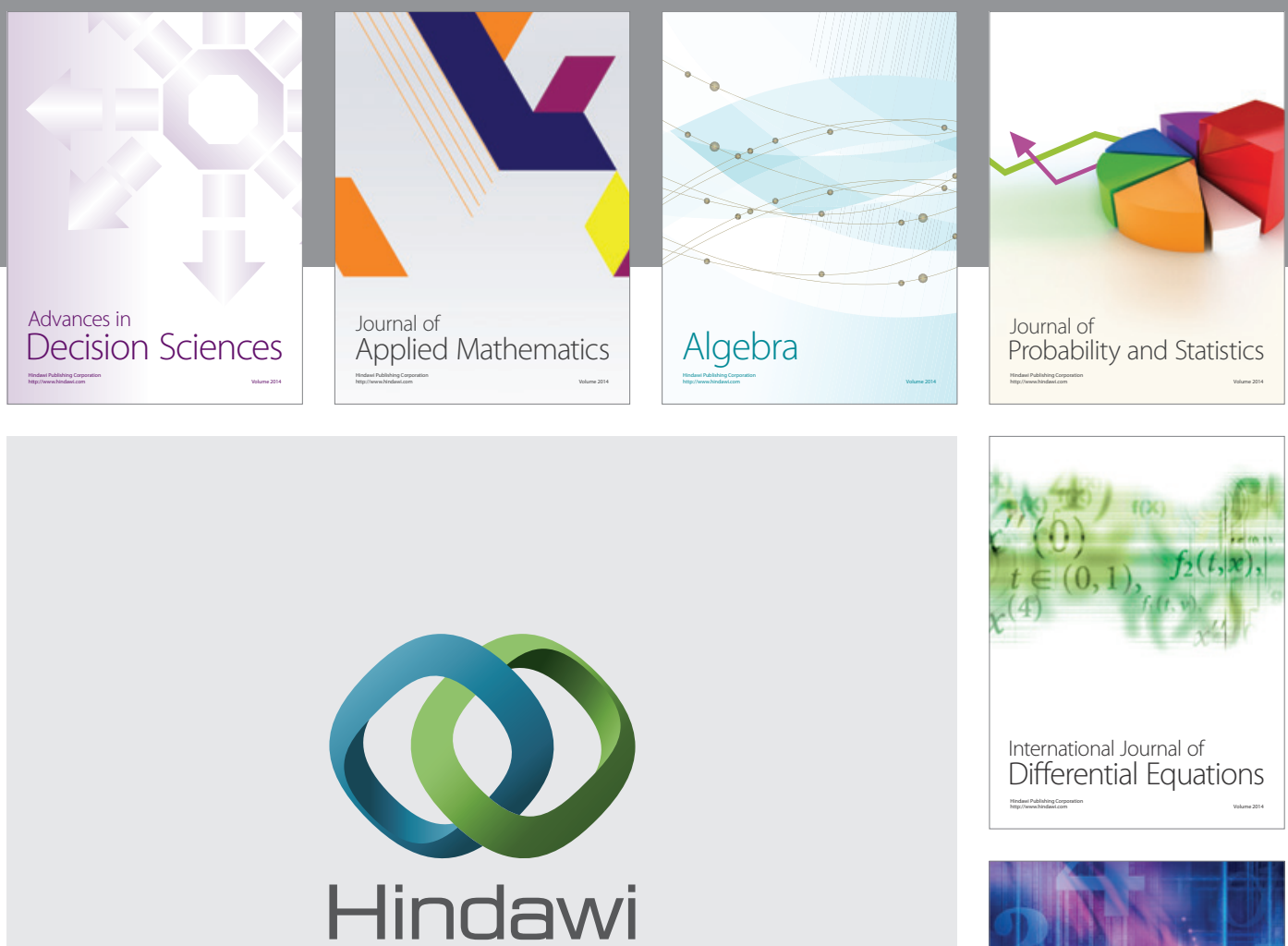

Submit your manuscripts at http://www.hindawi.com
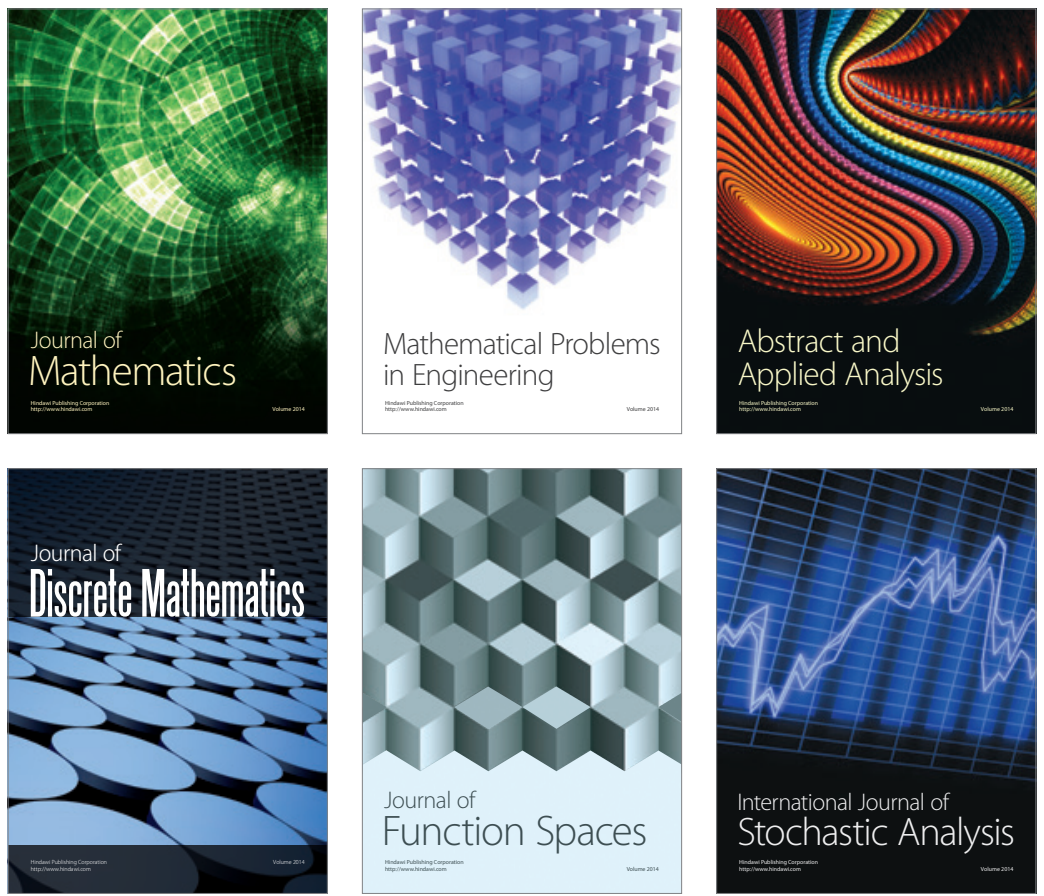

Journal of

Function Spaces

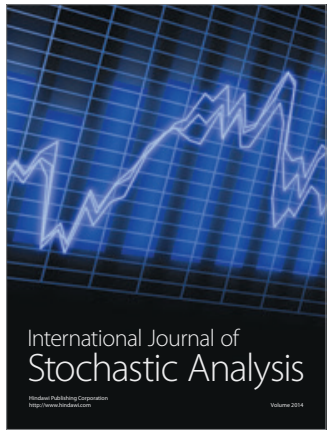

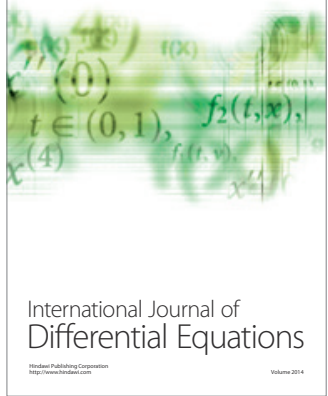
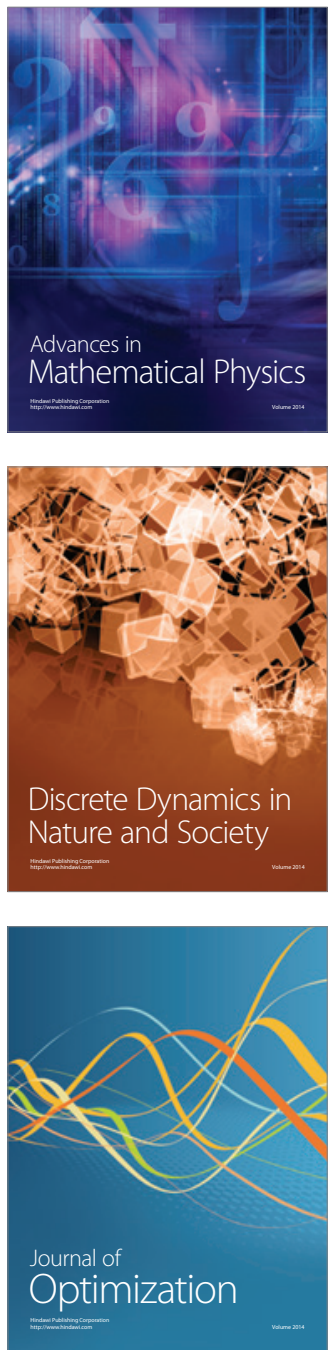